\title{
A Novel Approach for Effective Emotion Recognition Using Double Truncated Gaussian Mixture Model and EEG
}

\author{
Dr. N Murali Krishna \\ Associate Professor, Department of Computer Science and Engineering, \\ Vignan Institute of Technology and Science, JNTU (H) India \\ E-mail: muralinamana@gmail.com \\ Dr. J Sirisha Devi \\ Associate Professor, Department of Computer Science and Engineering, \\ Institute of Aeronautical Engineering, JNTU (H) India \\ E-mail: siri.cse21@gmail.com \\ Dr. Srinivas Yarramalle \\ Professor, Department of IT, GITAM University, India \\ E-mail: hod_it@gitam.edu
}

\begin{abstract}
Most of the models projected in the literature on Emotion Recognition aims at recognizing the emotions from the mobilized persons in noise free environment and is subjected to the emotion recognition of an individual using a single word for testing and training. Literature available to identify the emotions in case of immobilized persons is confined to the results available from the machines only. In this process braincomputer interaction is utilized using neuro-scan machines like Encephalography (EEG), to identify the emotions of immobilized individuals. It uses the physiological signals available from EEG data extracted from the brain signals of immobilized persons and tries to determine the emotions, but these results vary from machine to machine, and there exists no standardization process which can identify the feelings of the brain diseased persons accurately. In this paper a novel method is proposed, Doubly Truncated Gaussian Mixture Model (DT-GMM) to have a complete emotion recognition system which can identify emotions exactly in a noisy environment from both the healthy individuals and sick persons. The results of the proposed system surpassed the accuracy rates of traditional systems.
\end{abstract}

Index Terms-Emotion recognition, Doubly Truncated Gaussian Mixture Model, Encephalography.

\section{INTRODUCTION}

Human-computer interaction is possible area of interest since the birth of computer era. The strides of computer interaction can be witnessed over the years from punch cards systems to keyboards and mouse. These developments in the human-computer interaction are mainly focused towards the design of a user-friendly and ergonomic system which is most efficient, and communication can be done with at most comfort [1]. These technologies are embedded with interfaces such as voice recognition, face recognition.

Technological updates help to offer a direct humancomputer interaction employing signals which are generated from the brain. These methodologies contribute to serve the people having Neuro-muscular disabilities, thereby helping them in recognition the people. These technologies, as it is known as braincomputer interaction, facilitate to control the application of the equipment using the signals generated by the brain during the thought process [2] [3].

The developments in the area of cognitive neuroscience have been instigated latest advancements in the field of brain-computer interaction in particular to brain image technology. The Electroencephalography (EEG) [4], functional magnetic imaging (fMRI), Magneto Encephalography (MEG) are mainly used for brain analysis among these technologies EEG, can be acquired cheaply and it is noninvasive which helps to identify the functional correlations in the brain. The EEG plays a critical as well as a vital role in recognition of the ideas actually from the immobilized persons. These thoughts (or) emotions play a fundamental or major role in understanding the inherent feelings of the other person. Emotions act as a stimulus that evaluates the other persons based on the experiences. The emotions generated from the mobilized persons can be easily interpreted and various methodologies exist in the literature to identify the emotions of the individual [8].

However, these emotions that are generated from mobilized persons are from acting sequences, where as in order to have the exact emotions of the individual, it is necessary to understand and interpret the thoughts using the brain signals for which EEG will be more effective. 
The emotion recognition models that are presented in the earlier chapter may be restricted to mobilized persons only. To interact the emotions of immobilized individuals it necessary to estimate and evaluate the brain signals actually. This interpretation of the signal may be very much useful to understand the feelings of immobilized persons [6]. In particular brain disease, where the effectiveness of drug during the treatment can be identified by reading the brain emotions. The emotions are categorized into four major groups as in the case of mobilized persons viz, happy, sad, angry and neutral. If the brain signals from the EEG are interpreted as happy, in case of immobilized during the treatment in hospital, implies that he/ she is responding to the drug, and comfort level can be expressed via happy emotions. Therefore to interpret these signals, it is very much necessary to identify the brain signals effectively [7]. Hence in this paper we propose a novel approach using Truncated Gaussian Mixture Model to identify the brain signals whereby the emotions can be classified and the treatment can be addressed.

Outline of the remaining paper, section -2 gives a brief review on research work so far done for emotion recognition using EEG. Section -3 presents the gap observed in the research area based on the previous section. Section -4 presents the proposed novel approach. Section -5 presents the proposed system. Section- 6 gives details on experimentation and results obtained. Section 7 presents the conclusion.

\section{RELATED WORKS ON EMOTION RECOGNITION USING EEG}

Shuang Liu et al. (2016) used Power spectral density (PSD) and brain asymmetry (BAY) from EEG features and Support vector machine classifier. They were able to achieve $93.31 \%$ of accuracy rate for PSD and $85.39 \%$ with BAY. Tanfang Chen et.al. (2016) tried to recognize the emotions using DRBM by building the hidden relationship among the user profiles, EEG and emotion labels. Aravind E. Vijayan et.al (2015) used statistically weighed autoregressive modeling of Electroencephalogram (EEG) signals for emotion recognition and were able to achieve an accuracy of $94.097 \%$.

Priyanka Abhang et al. (2011) have proposed a paper which specifies the used EEG brain signals and speech in various languages to recognize human emotions. Methods used are Emotion tracking and Higher Order crossings and Empirical Mode Decomposition. Techniques used for feature extraction are Independent Component Analysis (ICA), Mel frequency cepstral coefficient (MFCC), KNN method, and wavelet. Panagiotis C. et al (2010) have proposed a paper in which they have concentrated on recognizing the emotion based on EEG feature extraction and the basic concept neuron system was introduced. Here they consider a feature extraction scheme like higher order crossings analysis is active as well as robust classification method, it call as HOC-emotion classifier (HOC-EC), it was achieve testing four different classifiers in order to accomplish efficient emotion identification. Results include QDA has obtained $62.3 \%$ mean successful rate, for the mixture channel case, best results are obtained by SVM $(83.33 \%$ mean classification rate).

Murugappan el at (2010) in their paper identified the human emotions with the help of wavelet transform using EEG signals. Here they are considering five different emotions namely natural, happy, fear, disgust, and surprise. 10-10 system which is having 64 electrodes is taken to collect EEG signals. Preprocessing is done with the help of SL filter. They are classifying the emotions with the help of DTW. KNN and LDA are utilized for performance evaluation and emotional state classification The accuracy rate is $83.26 \%$. Reza Khosrowabadi et al (2010) proposed a paper focuses on classification of EEG have shown a relationship on emotions with the help of features extracted GM of EEG spectrogram. Fractional dimension of EEG signal is compared with three feature extraction methods, for features extraction they are using KNN and SVM .In this paper they have investigated four different emotions, which include positive, negative calm and excited. Finally the features are extracted by using the Gaussian mixtures of EEG spectrogram. Ali S. AlMejrad (2010) has proposed a paper proposed a real time system to recognize the emotions with the help of EEG and they have discussed sensitivity activities in point of EEG measurement and noise removal and artifacts. They have extracted features with the help of wavelet transform and they have used different classification methods like SVM, Radial basis Function, Genetic Algorithm, Linear Discriminant Analysis.

M Murugappan et al (2010) proposed a paper focusing mainly on the people who are physically immobilized. They tried to establish a new communication medium for the immobilized people. Here they have adopted international standard video clips for identifying human emotions like happy disgust, fear and surprise. With the help of wavelet functions they extracted the statistical features of human brain signals (EEG). "Fuzzy C-Means (FCM) clustering" was used for the classification of emotion. The conclusion in this paper shows an encouraging result for detection of emotion. Murugappan.M et al (2009) in his paper anticipated a competent gaining protocol for gathering the EEG signals. For the pre-processing AMR method was used. For experimentation, they are taken six healthy people and 63 biosensors system is utilized. EEG parameters are extracted from discrete wavelet transform before we need to pre-process the signals. Using Fuzzy C-Means (FCM) clustering for the emotional classification purpose they derived Statistical features named as discrete emotions.

After a brief study of literature we come to a conclusion that very sparse research is done towards emotion recognition from EEG for immobilized persons. Hence in this paper an attempt is made to identify the emotional state of brain diseased persons. 


\section{GAP OBSERVED}

The emotions are to be extracted from the immobilize persons, Nero Scans will be much useful, for which Electroencephalography (EEG) will be more useful, which refers to the measures of brain electric activity [9] [10]. The measurement of the brain electrical activity can be obtained by the placing the EEG electrode on the brain scalp. Several works have been reported in literature based on the physiological signals such as EEG, ECG, Skin conductivity, EMG and Heart Rate. Most of the literature available regarding the brain diseased persons / immobilized persons is subjected to the movement of the arm position or eye movements and no research is driven to understand the emotions of the immobilized persons, hence this paper is further extended to study the emotions by developing two different models and extracting the brain signals using the EEG [11]. The initial estimates of the model parameters of the Doubly Truncated Gaussian Mixture Model are updated using the EM algorithm. The emotions that are generated by the human brain are interpreted from the EEG signals using the limbic system. Various regions in the brain, that contribute in assessing the emotions include,

1. Left Temporal areas: sadness.

2. Right Prefrontal Cortex area: sadness, happiness and disgust.

3. Bilateral Temporal activation: fear.

Apart from the above, all emotions share the areas viz., prefrontal cortex, cingulated gyrus and temporal cortex. In order to obtain the EEG signals, the subjects are to be trained, if mentally fit, then explained about the system positioning of the electrodes. Time duration should have a significant role in the extraction process; process should be done in the processes of trained technician. Fp1, FpZ, Fp2, F7, F3, F2, F4 and F8 electrodes are utilized to recognize the emotions. The brain signals from the diseased persons are extracted using the EEG, and the signals are preprocessed to minimize the noise and the amplitude signals are extracted which are normalized into different ranges basing on the rhythm, for the dimensionality reduction [13] [14]. These features extracted are modeled using the Doubly Truncated Gaussian Mixture Model and the Probability Density function of each rhythm is extracted, for each of the emotions that are exhibited the subject, the Probability density functions are calculated and basing on the Maximum likelihood estimate of each signal, the emotions are classified [15] [16].

\section{Proposed Model: Double TrunCATEd Gaussian MIXTURE MODEL}

Using Doubly Truncated Gaussian Distribution Model is that, the infinite range of the EEG samples can be truncated between a certain limits and the recognition of the emotions can be carried out using the finite range as shown in figure 1 , figure 2 and figure 3.

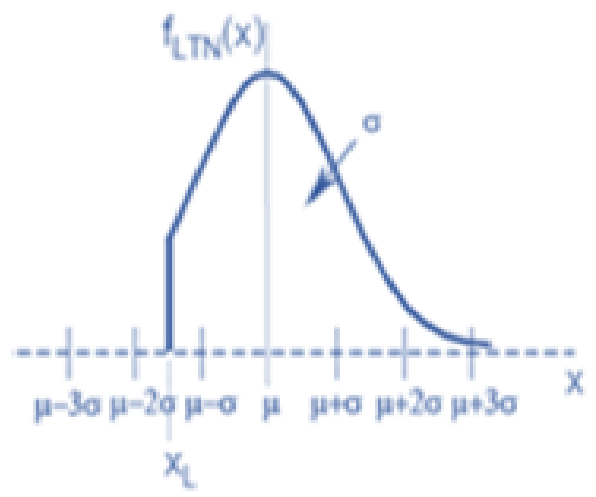

Fig.1. Left Truncated Normal Distribution

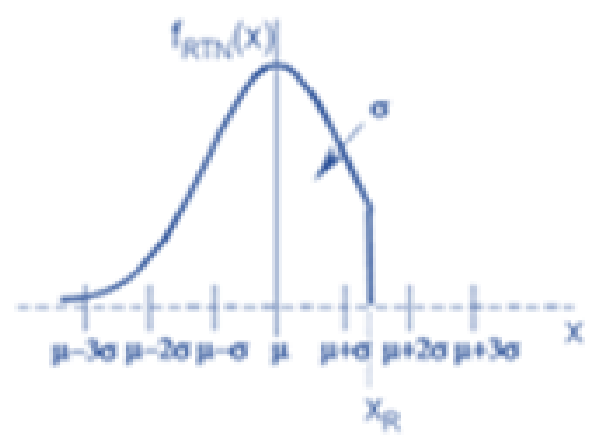

Fig.2. Right Truncated Normal Distribution

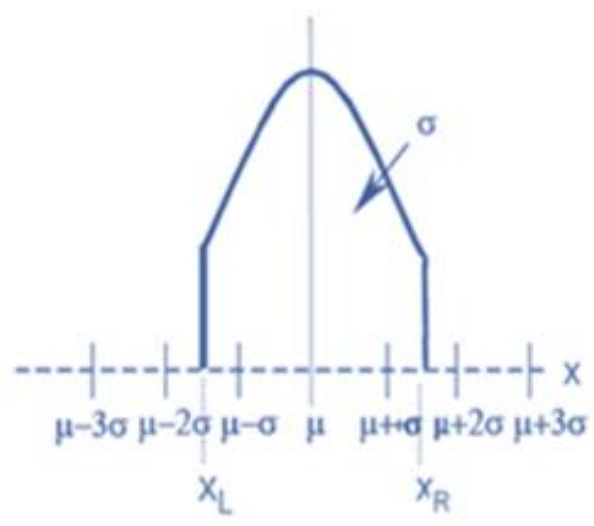

Fig.3. Doubly-Truncated Normal Distribution

A. The Probability Density Function (PDF) of GMM

The PDF of GMM is given by equation (1)

$$
f(z)=\frac{1}{\sqrt{2 \pi \sigma}} e^{\frac{-1}{2}\left(\frac{z-\mu}{\sigma}\right)^{2}} ;-\infty<z<+\infty, 0<\sigma
$$

The value of $\mathrm{Z}$ below some $\mathrm{Z}_{\mathrm{L}}$ and above some $\mathrm{Z}_{\mathrm{M}}$ cannot be possible to exist. Then the resulting distribution is a Doubly Truncated Gaussian Distribution and the PDF is given by equation (2) 


$$
g(z)=\frac{g(z)}{\int_{Z_{M}}^{Z_{\mathrm{L}}} f(z) d z},-\infty \leq z<\infty
$$

Where $Z_{L} \leq Z \leq Z_{M}$ and $Z_{M} \leq Z<\infty$

$$
\text { This implies } g(z)=\frac{f(z)}{B-A}, Z_{L}<Z<Z_{M}
$$

Where,

$$
A=\int_{-\infty}^{Z_{L}} \frac{e^{\frac{-1}{2}\left(\frac{z-\mu}{\sigma}\right)^{2}}}{\sqrt{2 \pi \sigma}} d z \text { and } B=\int_{-\infty}^{Z_{M}} \frac{e^{\frac{-1}{2}\left(\frac{z-\mu}{\sigma}\right)^{2}}}{\sqrt{2 \pi \sigma}} d z
$$

Therefore,

$$
g(z)=\frac{\frac{1}{\sqrt{2 \pi \sigma}} e^{\frac{-1}{2}\left(\frac{z-\mu}{\sigma}\right)^{2}}}{\int_{-\infty}^{M} \frac{1}{\sqrt{2 \pi \sigma}} e^{\frac{-1}{2}\left(\frac{z-\mu}{\sigma}\right)^{2}} d z-\int_{-\infty}^{Z_{L}} \frac{1}{\sqrt{2 \pi \sigma}} e^{\frac{-1}{2}\left(\frac{z-\mu}{\sigma}\right)^{2}} d z}
$$

The lower and upper truncation points are $\mathrm{Z}_{\mathrm{L}}$ and $\mathrm{Z}_{\mathrm{M}}$ respectively.

B. Estimation of the model parameter using ExpectationMaximization Algorithm

The likelihood function of sample observation $\left\{Z_{1}, Z_{2}, \ldots, Z_{n}\right\}$ drawn with the Probability Density Function $h(z, \theta)=\sum \alpha_{i} g_{i}\left(z_{s}, \theta\right)$ is given in equation (5)

$$
\begin{aligned}
& L(\theta)=\pi_{s=1}^{N}\left(\sum_{l=1}^{k} \alpha_{i} g_{i}\left(z_{s}, \theta\right)\right) \\
= & \pi_{s=1}^{N}\left[\sum_{i=1}^{k} \alpha_{i} \frac{1}{\sqrt{2 \pi \sigma}(B-A)} e^{\frac{-1}{2}\left(\frac{z-\mu}{\sigma}\right)^{2 ?}}\right]
\end{aligned}
$$

Where $\mathrm{A}$ and $\mathrm{B}$ are given above.

$$
\begin{gathered}
\log (\theta)=\log \left(\sum_{k=1}^{k} \alpha_{i} g_{i}\left(z_{s}, \theta\right)\right) \\
=\sum_{s=1}^{N} \log \left(\sum_{i=1}^{k} \alpha_{i} g_{i}\left(z_{s}, \theta\right)\right)
\end{gathered}
$$

E step:

$$
Q\left(\theta, \theta^{(0)}\right)=E_{\theta}^{(0)}\{\log L(\theta) / \bar{z}\}
$$

Given the initial parameters $\theta^{l}$, one cam computes the density of any observation $Z_{s}$ as

$$
\begin{array}{r}
h\left(Z_{s} ; \theta\right)=\sum_{i=1}^{k} \alpha_{i}^{l} g_{i}\left(Z_{s}, \theta^{l}\right) \\
=\pi_{s-1}^{N}\left[\sum_{i=1}^{k} \alpha_{i} \frac{\frac{-1}{2}\left(\frac{Z-M_{i}}{\sigma_{i}}\right)^{2}}{\sqrt{2 \pi} \xi(B-A)}\right]
\end{array}
$$

The conditional probability of any observation $Z_{j}$ to belong to the segment $\mathrm{k}$ as

$$
t_{k}\left(Z_{s} ; \theta^{l}\right)=\frac{\alpha_{k}^{l} g_{i}\left(Z_{s}, \theta^{l}\right)}{h\left(Z_{s} ; \theta^{l}\right)}=\frac{\alpha_{k}^{l} g_{k}\left(Z_{s}, \theta^{l}\right)}{\sum_{i=1}^{k} \alpha_{k}^{l} g_{i}\left(Z_{s}, \theta^{l}\right)}
$$

Since $h\left(Z_{s} ; \theta^{l}\right)=\sum_{i=1}^{k} \alpha_{k}^{l} g_{k}\left(Z_{s}, \theta^{l}\right)$ from equation

$$
c=\frac{\sqrt{\operatorname{var}(z)}}{E(Z)}
$$

Evaluating the expectation value $L(\theta)$ is

$$
Q\left(\theta ; \theta^{l}\right)=E_{\theta^{l}}\{\log L(\theta)\}
$$

Following the heuristic arguments of Jeff. A Bilmes (1997) we get

$$
Q\left(\theta ; \theta^{l}\right)=\sum_{i=1}^{k} \sum_{s=1}^{N} E^{l}\left\{t_{i}\left(Z ; \theta^{l}\right)\left(\log g_{i}(Z, \theta)+\log \alpha_{i}\right)\right\}
$$

But we have $g_{i}(z ; \theta)=\frac{f_{i}(z, \theta)}{B-A}$

Since here the distribution is truncated.

Where $f_{i}(z, \theta)=\frac{1}{\sqrt{2 \pi \sigma}} e^{\frac{-1}{2}\left(\frac{z-\mu}{\sigma}\right)^{2}}$

Therefore,

$$
Q\left(\theta, \theta^{(l)}\right)=\sum_{i=1}^{k} \sum_{s=1}^{N} E^{(l)}\left\{t_{i}\left(z, \theta^{l}\right)\left(\log g_{i}(z ; \theta)+\log \alpha_{i}\right)\right\}
$$

Substitute the value of $\log g_{i}(z ; \theta)$ we have

$$
\sum_{t=1}^{s} \sum_{s=1}^{N} E^{(l)}\left\{t_{i}\left(z, \theta^{l}\right)(\log f(z ; \theta)-\log (B-A))+\log \alpha_{\mathrm{i}}\right\}
$$

M- Step: The problem of calculating the maximum likelihood estimation of the segment weight $\alpha_{k}$, under 
the additional condition $\sum_{i=1}^{k} \alpha_{i}=1$ can be solved by applying the standard solution for constrained maximum. We construct the first order Lagrange-type function.

$$
L=E^{(l)}\left[\log L\left(\theta^{l}\right)+\lambda\left(1-\sum_{i=1}^{k} \alpha_{i}^{l}\right]\right.
$$

Where $\lambda$ is a Lagrangian multiplier combining constraints with log likelihood function to be maximized. The derivative of $\mathrm{L}$

$$
\frac{\partial L}{\partial \alpha_{k}}=0 \Rightarrow \frac{\partial}{\partial \alpha_{k}}\left(E^{(l)}\left[\log L\left(\theta^{l}\right)+\lambda\left(1-\sum_{i=1}^{k} \frac{\partial L}{\partial \alpha_{k}}\right]\right)=0\right.
$$

This implies

$$
\begin{gathered}
\sum_{s=1}^{N}\left[\frac{\sum_{i=1}^{k} E^{(l)}\left[\frac{\partial\left(\alpha_{i}^{l} g_{i}\left(z_{s}, \theta^{l}\right)\right)}{\partial \alpha_{k}}\right]}{\sum_{i=1}^{k} \alpha_{i}^{l} g_{i}\left(z_{s}, \theta^{l}\right)}\right]-\lambda=0 \\
\Rightarrow \sum_{i=1}^{k} E^{(l)}\left[\frac{\left.g_{k}\left(z_{s}, \theta^{l}\right)\right)}{h\left(z_{s}, \theta^{l}\right)}\right]-\lambda=0
\end{gathered}
$$

Multiplying and dividing by $\alpha_{k}$ we have $\frac{1}{\alpha_{k}} \sum_{s=1}^{N} \frac{\left.E^{l} \alpha_{k} g_{k}\left(z_{s}, \theta^{l}\right)\right)}{h\left(z_{s}, \theta^{l}\right)}-\lambda=0$

This implies

$$
\frac{1}{\alpha_{k}} \sum_{s=1}^{N} E^{l}\left(t_{k}\left(z_{s}, \theta^{l}\right)\right)-\lambda=0
$$

Where

To update the estimates of $\alpha_{k}^{l}$ we have to go to the next level, i.e, $\alpha_{k}^{l+1}$ we have,

$$
\frac{1}{\alpha_{k}} \sum_{s=1}^{N} E^{l}\left(t_{k}\left(z_{s}, \theta^{l}\right)\right)=\lambda
$$

This implies

$$
\sum_{s=1}^{N} E^{l}\left(t_{k}\left(z_{s}, \theta^{l}\right)\right)=\lambda \alpha_{k}
$$

Taking sum all over $\mathrm{k}=1,2,3 \ldots \mathrm{k}$ and considering that for every $z_{s}$, the sum over all $t_{k}\left(z_{s}, \theta^{l}\right)$ must be equal to ONE, due to the probability character of $t_{k}\left(z_{s}, \theta^{l}\right)$ we get

$$
\sum_{i=1}^{k} \sum_{s=1}^{N} t_{i}\left(z_{s}, \theta^{l}\right)=\lambda=N
$$

$$
\text { Therefore } \alpha_{k}^{l+1}=\frac{1}{N} \sum_{s=1}^{N} E^{l}\left\{t_{k}\left(Z_{s}, \theta^{l}\right)\right\}
$$

We have

$$
E^{l}\left\{t_{k}\left(Z_{s}, \theta^{l}\right)\right\}=\int_{Z_{L}}^{Z_{M}} \alpha_{k}^{l} \frac{g_{k}\left(Z_{s}, \theta^{l}\right)}{h\left(Z_{s}, \theta^{l}\right)} \frac{h\left(Z_{s}, \theta^{l}\right) d z}{\int_{Z_{L}}^{Z_{M}} h\left(Z_{s}, \theta^{l}\right) d z}
$$

Therefore $\alpha_{k}^{l+1}=\frac{1}{N} \sum_{s=1}^{N} \frac{\alpha_{k}^{l}}{H\left(Z_{M}, \theta^{l}\right)-H\left(Z_{L}, \theta^{l}\right)}$

where

$$
H\left(Z_{M}, \theta^{l}\right)=\int_{-\infty}^{Z_{M}} \alpha_{i} g_{i}\left(Z_{s}, \theta^{l}\right) d z
$$

and

$H\left(Z_{L}, \theta^{l}\right)=\int_{-\infty}^{Z_{L}} \alpha_{i} g_{i}\left(Z_{s}, \theta^{l}\right) d z$

For updating $\mu$ : for updating the parameters, $\mu_{k}$, $\mathrm{k}=1,2, \ldots \mathrm{k}$. we consider the derivation of $Q\left(\theta, \theta^{l}\right)$ with respect to $\mu_{k}$ equated to zero.

$$
\frac{\partial Q\left(\theta, \theta^{l}\right)}{\partial \mu_{k}}=0
$$

$\Rightarrow \frac{\partial}{\partial \mu_{k}} \sum_{i=1}^{k} \sum_{s=1}^{N} E^{(l)}\left\{t_{i}\left(z_{s}, \theta^{l}\right)\left(\log g_{i}(z ; \theta)\right)+\log \alpha_{i}\right\}=0$

By taking expectations from equation (10)

$$
\sum_{s=1}^{N} E^{(l)}\left\{t_{i}\left(z_{s}, \theta^{l}\right) \frac{\partial}{\partial \mu_{k}}\left(\log g_{i}(z ; \theta)\right)\right\}=0
$$

But we have 
$$
\left(\log g_{k}(z ; \theta)\right)=\log \left[\frac{\frac{1}{\sqrt{2 \pi \sigma}} e^{\frac{-1}{2}\left(\frac{z-\mu_{k}}{\sigma_{k}}\right)^{2}}}{\int_{z_{l}}^{z_{m}} \frac{1}{\sqrt{2 \pi \sigma}} e^{\frac{-1}{2}\left(\frac{z-\mu_{k}}{\sigma_{k}}\right)^{2}} d z}\right]
$$

And

$\frac{\partial}{\partial \mu_{k}}\left(\log g_{k}(z ; \theta)\right)=$

$\frac{\partial}{\partial \mu_{k}}\left(-\log \sqrt{2 \pi}-\log \sigma_{k}-\frac{1}{2}\left(\frac{z-\mu_{k}}{\sigma_{k}}\right)^{2}-\log \int_{z_{l}}^{z_{m}} \frac{1}{\sqrt{2 \pi \sigma}} e^{\frac{-1}{2}\left(\frac{z-\mu_{k}}{\sigma_{k}}\right)^{2}} d z\right)$

This implies that

$$
\frac{\partial}{\partial \mu_{k}}\left(\log g_{k}(z ; \theta)\right)=\frac{1}{\sigma_{k}^{2}}\left(z-\mu_{k}\right)-\frac{\int_{z_{l}}^{z_{m}} \frac{\partial}{\partial \mu_{k}} f_{k}(z, \theta) d z}{\int_{z_{l}}^{z_{m}} f_{k}(z, \theta) d z}
$$

We have $\frac{\partial}{\partial \mu_{k}} f_{k}\left(z, \theta^{l}\right)=\left(\frac{z-\mu_{k}}{\sigma_{k}{ }^{2}}\right) f_{k}\left(z, \theta^{l}\right)$

This implies

$$
\sigma_{k}^{2} \frac{\partial}{\partial \mu_{k}} f_{k}\left(z, \theta^{l}\right)=z f_{k}\left(z, \theta^{l}\right)-\mu_{k}^{l} f_{k}\left(z, \theta^{l}\right)
$$

This implies

$$
z f_{k}\left(z, \theta^{l}\right)=\mu_{k} f_{k}\left(z, \theta^{l}\right)+\sigma^{2} \frac{\partial}{\partial \mu_{k}} f_{k}\left(z, \theta^{l}\right)
$$

The second part of the equation

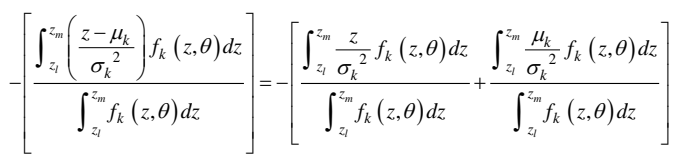

Since $\frac{\int_{z_{l}}^{z_{m}} z f_{k}(z, \theta) d z}{\int_{z_{l}}^{z_{m}} f_{k}(z, \theta) d z}=\mu^{l}+\sigma^{2}\left(\frac{f\left(z_{m}\right)-f\left(z_{l}\right)}{B-A}\right)$ and $\frac{\int_{z_{l}}^{z_{m}} \frac{\mu_{k}}{\sigma_{k}^{2}} f_{k}(z, \theta) d z}{\int_{z_{l}}^{z_{m}} f_{k}(z, \theta) d z}=\frac{\mu_{k}}{\sigma_{k}^{2}}$

Therefore

$$
-\left[\frac{\int_{z_{i}}^{z_{i}} \frac{z-\mu_{k}}{\sigma_{k}^{2}} f_{k}(z, \theta) d z}{\int_{z_{i}}^{m_{z}} f_{k}(z, \theta) d z}\right]=-\left[\frac{\mu_{k}^{\prime}}{\sigma_{k}^{2}} \frac{\sigma_{k}^{2}}{\sigma_{k}^{2}}\left(\frac{f\left(z_{m}\right)-f\left(z_{1}\right)}{B-A}-\frac{\mu_{k}^{\prime}}{\sigma_{k}^{2}}\right]=\left(\frac{f\left(z_{m}\right)-f\left(z_{1}\right)}{B-A}\right)\right.
$$

The equation becomes

$$
\frac{\partial}{\partial \mu_{k}}\left(\log g_{k}(z ; \theta)\right)=\frac{1}{\sigma_{k}^{2}}\left(z-\mu_{k}\right)+\left(\frac{f\left(z_{m}\right)-f\left(z_{l}\right)}{B-A}\right)
$$

This implies

$$
\Rightarrow \quad \frac{\partial}{\partial \mu_{k}}\left(\log g_{k}(z ; \theta)\right)=\frac{\left(z-\mu_{k}\right)+\left(\frac{f\left(z_{m}\right)-f\left(z_{l}\right)}{B-A}\right) \sigma_{k}^{2}}{\sigma_{k}{ }^{2}}
$$

There from equation 24 , we have

$$
\frac{\sum_{s=1}^{N} E^{(l)}\left\{t_{k}\left(z_{s}, \theta^{l}\right)\left(z-\mu_{k}\right)-\left(\frac{f\left(z_{m}\right)-f\left(z_{l}\right)}{B-A}\right) \sigma_{k}^{2}\right\}}{\sigma_{k}^{2}}=0
$$

This implies

$$
\mu_{k}=\frac{\sum_{s=1}^{N} E^{(l)}\left\{\left[t_{k}\left(z_{s}, \theta^{l}\right) z\right]+\sum_{s=1}^{N} t^{k}\left(z_{s}, \theta^{l}\right)\left(\frac{f\left(z_{m}\right)-f\left(z_{l}\right)}{B-A}\right) \sigma_{k}^{2}\right\}}{\sum_{s=1}^{N} E^{(l)} t_{k}\left(z_{s}, \theta^{l}\right)}=0
$$

The first term of the equation is

$$
\begin{gathered}
E^{(l)}\left[t_{k}\left(z_{s}, \theta^{l}\right) z\right]=\int_{z_{l}}^{z_{m}} \frac{z \alpha_{k}^{l} g_{k}\left(z_{s}, \theta^{l}\right)}{z_{z_{l}} h\left(z_{s}, \theta^{l}\right) d z} d z \\
=>\frac{\alpha_{k}{ }^{l}\left[\mu_{k}^{l}+\sigma_{k}^{2}\left(\frac{f\left(z_{m}\right)-f\left(z_{l}\right)}{B-A}\right)\right]}{\int_{z_{l}}^{z_{m}} h\left(z_{s}, \theta^{l}\right) d z}
\end{gathered}
$$

The second term of equation is

$$
\begin{aligned}
& \sigma_{k}^{2}\left(\frac{f\left(Z_{M}\right)-f\left(Z_{L}\right)}{B-A}\right)_{Z_{L}}^{Z_{M}} \frac{\alpha_{k}^{l} g_{k}\left(Z_{s}, \theta^{l}\right) d z}{\int_{Z_{L}}^{Z_{M}} h\left(Z_{s}, \theta^{l}\right) d z} \\
& =\alpha_{k}^{l} \sigma_{k}^{2}\left(\frac{f\left(Z_{M}\right)-f\left(Z_{L}\right)}{B-A}\right) \frac{1}{\int_{Z_{L}}^{Z_{M}} h\left(Z_{s}, \theta^{l}\right) d z}
\end{aligned}
$$


The denominator of the equation (35) can be simplified as

$$
E^{l}\left\{t_{k}\left(Z_{s}, \theta^{l}\right)\right\}=\frac{\alpha_{k}^{l}}{\int_{Z_{L}}^{Z_{M}} h\left(Z_{s}, \theta^{l}\right) d z}
$$

Hence

$$
\mu_{k}^{l+1}=\frac{\alpha_{k}^{l}\left[\frac{\mu_{k}^{l}+2 \sigma_{k}^{2 l}\left(\frac{f\left(Z_{M}\right)-f\left(Z_{L}\right)}{B-A}\right)}{\int_{Z_{L}}^{Z_{M}} h\left(Z_{s}, \theta^{l}\right) d z}\right]}{\frac{\alpha_{k}^{l}}{\int_{Z_{L}}^{Z_{M}} h\left(Z_{s}, \theta^{l}\right) d z}}=\mu_{k}^{l}+2 \sigma_{k}^{2 l}\left(\frac{f\left(Z_{M}\right)-f\left(Z_{L}\right)}{B-A}\right)
$$

For updating $\sigma_{k}^{2}$ :

For updating $\sigma_{k}^{2}$ we consider the derivative of $Q\left(\theta, \theta^{l}\right)$ with respect to $\sigma_{k}^{2}$ and equate it to zero.

$$
\begin{aligned}
& \frac{\partial}{\partial \sigma_{k}^{2}} Q\left(\theta, \theta^{l}\right) \\
= & \frac{\partial}{\partial \sigma_{k}^{2}} \sum_{i=1}^{k} \sum_{s=1}^{N} E^{(l)}\left\{t_{i}\left(z_{s}, \theta^{l}\right)\left(\log f_{i}(z ; \theta)\right)+\log \alpha_{i}\right\} \\
= & \sum_{s=1}^{N} E^{(l)}\left\{t_{i}\left(z_{s}, \theta^{l}\right) \frac{\partial}{\partial \sigma_{k}^{2}}\left(\log f_{k}(z ; \theta)\right)\right\}
\end{aligned}
$$

This implies

$$
\sum_{s=1}^{N}(l)\left\{\begin{array}{c}
t_{k}\left(z_{s}, \theta\right) \frac{\partial}{\partial \sigma_{k}^{2}}\left(\log \sqrt{2 \pi}-\log \sigma_{k}-\frac{1}{2}\left(\frac{z-\mu_{k}}{\sigma_{k}}\right)^{2}\right) \\
-\log \int_{z_{l}}^{z_{m}} f_{k}(z ; \theta) d z+\log \alpha_{k}
\end{array}\right\}
$$$$
=\sum_{s=1}^{N} E^{(l)}\left\{\begin{array}{l}
t_{k}\left(z_{s}, \theta\right)\left(\frac{1}{2 \sigma_{k}^{2}}\left(\frac{z-\mu}{\sigma_{k}^{2}}\right)-1\right) \\
-\frac{\int_{z}^{z_{m}} \frac{\partial}{\partial \sigma_{k}^{2}} f_{k}\left(z ; \theta_{k}\right) d z}{\int_{z l}^{z_{m}} f_{k}\left(z ; \theta_{k}\right) d z}
\end{array}\right\}
$$

The second part of the equation i.e,

$$
\frac{\int_{z_{l}}^{z_{m}} \frac{\partial}{\partial \sigma_{k}^{2}} f_{k}\left(z ; \theta_{k}\right) d z}{\int_{z_{l}}^{z_{m}} f_{k}\left(z ; \theta_{k}\right) d z}=\frac{\frac{1}{2} \int_{z_{l}}^{z_{m}} f_{k}\left(z ; \theta_{k}\right)\left(\frac{1}{\sigma_{k}^{2}}-\left(\frac{z-\mu}{\sigma_{k}^{2}}\right)\right) d z}{\int_{z_{l}}^{z_{m}} f_{k}\left(z ; \theta_{k}\right) d z}
$$

Therefore

$$
\begin{aligned}
& \frac{\int_{Z_{L}}^{Z_{M}} \frac{\partial}{\partial \sigma_{k}^{2}} f_{k}\left(z, \theta_{k}\right) d z}{\int_{Z_{L}}^{Z_{M}} f_{k}\left(z, \theta_{k}\right) d z} \\
& =\frac{-1}{2 \sigma_{k}^{2}}\left(\begin{array}{l}
\frac{1}{B-A}-1-\left(1+\mu_{k}\right)\left(\frac{f_{k}\left(Z_{L}, \theta^{l}\right)-f_{k}\left(Z_{M}, \theta^{l}\right)}{B-A}\right) \\
+\frac{Z_{M} f_{k}\left(Z_{M}, \theta^{l}\right)-Z_{L} f_{k}\left(Z_{L}, \theta^{l}\right)}{B-A}
\end{array}\right)
\end{aligned}
$$

Substitute equation (18) in equation (24) we get

$$
\begin{aligned}
& \frac{\partial Q\left(\theta, \theta^{l}\right)}{\partial \sigma_{k}^{2}} \\
& =\sum_{s=1}^{N} E^{l}\left(t_{x}\left(z, \theta^{l}\right)\right) \\
& {\left[\frac{1}{2 \sigma_{k}^{2}}\left(\frac{Z-\mu}{\sigma_{k}^{2}}\right)^{2}-\frac{1}{2 \sigma_{k}^{2}}+\frac{1}{2 \sigma_{k}^{2}}-\frac{1}{2 \sigma_{k}^{2}(B-A)}\right.}
\end{aligned}
$$$$
\left.+\frac{1+\mu_{k}}{2 \sigma_{k}^{2}}\left(\frac{f_{k}\left(Z_{L}, \theta^{l}\right)-f_{k}\left(Z_{M}, \theta^{l}\right)}{B-A}\right)-\frac{1}{2 \sigma_{k}^{2}} \frac{f_{k}\left(Z_{L}, \theta^{l}\right)-f_{k}\left(Z_{M}, \theta^{l}\right)}{B-A}\right]
$$

Let

$D=\frac{1}{B-A}\left[\left(1+\mu_{k}\right)\left(f_{k}\left(Z_{L}, \theta^{l}\right)-f_{k}\left(Z_{M}, \theta^{l}\right)\right)+\left(Z_{M} f_{k}\left(Z_{M}, \theta^{l}\right)-Z_{L} f_{k}\left(Z_{L}, \theta^{l}\right)\right)\right]$ Implies

$$
\begin{gathered}
\sum_{s=1}^{N} E^{l}\left(t_{x}\left(z, \theta^{l}\right)\right)\left[\left(\frac{Z-M}{2 \sigma_{k}^{4}}\right)^{2}-\frac{D}{2 \sigma_{k}^{2}}\right]=0 \\
\sum_{s=1}^{N} E^{l}\left(t_{x}\left(z, \theta^{l}\right)\right)\left(\frac{Z-M}{2 \sigma_{k}^{4}}\right)^{2}=\sum_{s-1}^{N} E^{l}\left(t_{x}\left(z, \theta^{l}\right) \frac{D}{2 \sigma_{k}^{2}}\right)
\end{gathered}
$$




$$
\Rightarrow \quad \sigma_{k}^{2(l+1)}=\frac{\sum_{s-1}^{N} E^{l}\left(t_{k}\left(Z, \theta^{l}\right)\right)\left(Z_{s}-\mu_{k}^{l+1}\right)^{2}}{D \sum_{s-1}^{N} E^{l}\left(t_{x}\left(Z, \theta^{l}\right)\right)}
$$

By expanding and we have

$$
\begin{aligned}
& \sigma_{k}^{2(l+1)} \\
& =\frac{1}{D}\left\{\alpha_{k}^{l} \mu_{k}^{2 l}+\left[\frac{f_{k}\left(Z_{M}, \theta^{l}\right)-f_{k}\left(z_{L}, \theta^{l}\right)}{f\left(Z_{M}, \theta^{l}\right)-f\left(z_{L}, \theta^{l}\right)}\right]\right. \\
& -\left(\alpha_{k}^{l} \sigma_{k}^{2 l}-\alpha_{k}^{l} \mu_{k}^{l} \sigma_{k}^{2 l}\right) \\
& -\alpha_{k}^{l} \sigma_{k}^{2 l}\left(\frac{Z_{M} f_{k}\left(Z_{M}, \theta^{l}\right)-Z_{L} f_{k}\left(Z_{L}, \theta^{l}\right)}{f_{k}\left(Z_{M}, \theta^{l}\right)-f_{k}\left(Z_{L}, \theta^{l}\right)}\right) \\
& -2 \mu_{k}\left[\frac{\alpha_{k}^{l} \mu_{k}^{(l+1)}}{f\left(Z_{M}, \theta^{l}\right)-f\left(Z_{L}, \theta^{l}\right)}-\frac{\alpha_{k}^{l} \sigma_{k}^{2 l}}{f\left(Z_{M}, \theta^{l}\right)-f\left(Z_{L}, \theta^{l}\right)}\right] \\
& \left.-\mu_{k}^{2(l+1)}\right\}
\end{aligned}
$$

For effective performance evaluation of the system, the recognition accuracy of the developed model is evaluated using one way classification by using ANOVA, Precision and \& Recall.

\section{PRoposed SySteM}

After acquiring the brain signals, the frequency of each of the signals is identified [5]. A 10-20 electrode system was considered for experimentation. Each subject is first trained about the system and a test acquisition is conducted; after he / she get acquainted with the system, the actual readings generated and the frequencies generated are noted. The electrodes positions Fp1, FpZ, Fp2, F7, F3, F2, F4 and F8 electrodes are considered. This electrode position denotes the frontal positions and the emotions can be well interpreted from these potions. These frequencies are trained using DT-GMM. The process to be followed for the identification of the emotions is presented in algorithm in section A.

\section{A. Algorithm for acquisition of brain signals using EEG}

The steps to the followed for the acquisition of brain signals are given:

Step - 1 Train the database

Step -2 Find the MU \& SIGMA values of the training database

Step -3 Find the Probability Density function of each and every emotional dataset
Step -4 Extract the EEG Brain signals say Test database Step -5 Find the MU \& SIGMA values of the Test database

Step -6 Find the PDFs of the Test data.

Step -7 Compare the Test data with the Train database and identify which emotion data does the EEG matches with the existing dataset.

Step -8 Displays the Emotion

\section{EXPERIMENTATION AND RESULTS}

We have considered 50 subjects for training and 25 subjects for testing purpose, emotions namely angry, sad, happy and neutral are considered for experimentation. A basic block diagram to analyze an EEG signal is shown in figure 4.

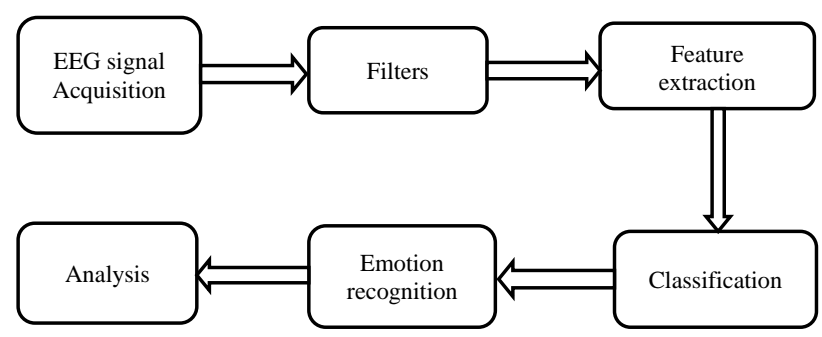

Fig.4. Basic block diagram to analyze the EEG signals

B. Methodology of interpreting the brain signals based on Truncated Gaussian Mixture Model for mobilized and immobilized people

In order to record the brain signals, a scalp is fixed on the brain recorded EEG the neuronal electrical activity is recorded non-invasively, typically using small metal plate electrodes. The recordings are done using reference electrode(s) or bipolar linkages; the number of the electrodes will be changes used from application to application, the electrodes are typically placed at specific scalp locations. The voltages, are of the order of microvolt's $(\mu \mathrm{V})$, reading should be carefully recorded to avoid interference and digitized so that it can be stored and viewed on a computer.

The general frame work of an EEG acquisition model is presented in figure 5 .

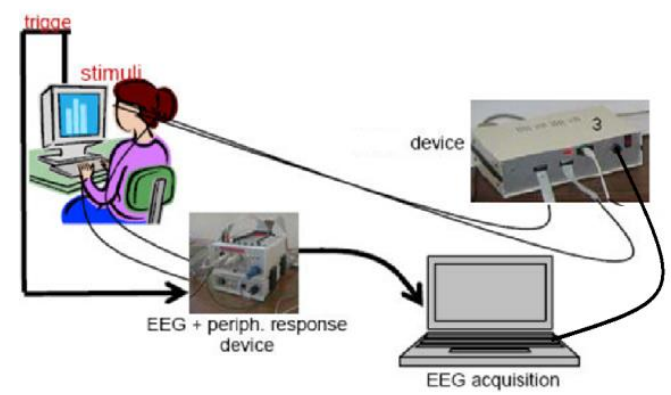

Fig.5. Hardware setup for signal acquisition

Prior to acquisition process, the electrodes will be in red color and when the electrodes are connected to the scalp using electro gel, these electrodes change their 
color to green; and if the connectivity is not established properly, it can be visible in a color other than green, generally grey. The typical framework is shown in figure 6.

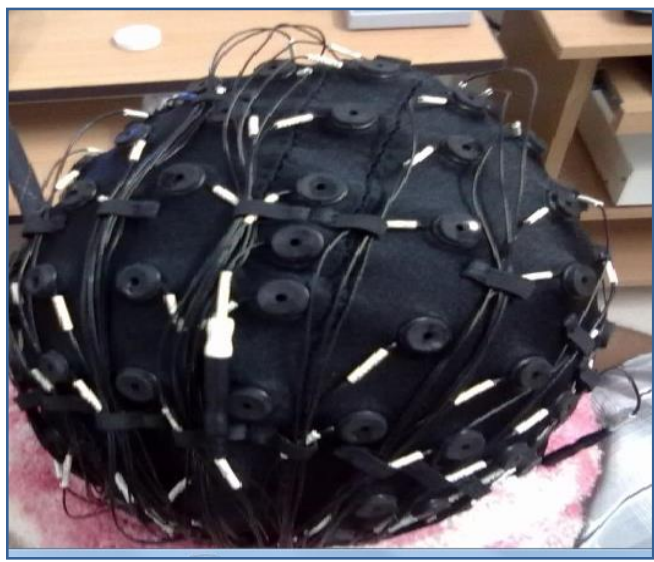

Fig.6. Participant wearing the EEG cap with 64 electrodes plugged

The basic EGG recording system consists of electrodes with conductive media, amplifiers with filters, an analogto-digital (A/D) converter and finally a recording device to store the data. Electrodes, in conjunction with the electrode gel, sense the signal from the scalp surface; amplifiers bring the microvolt and often nano volt signals into a range where they can be digitized accurately; and the A/D converter changes signals from analog to digital form that can be finally stored or viewed on a computer. The various electrodes used and their locations are presented in following figure 7 .

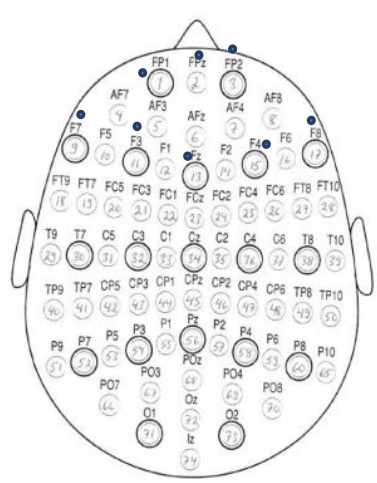

Fig.7. Top head view with the positions and names of the 64 electrodes used for EEG recording

For experimentation, eight electrodes configuration, only the BLUE electrodes were used. The electrode is placed with different positions such as 10-10 electrodes system, 10-20 electrode systems, 10-5 electrode systems according to the application. Dataset used for testing is SEED dataset and DEAP dataset. The experimentation with DT-GMM is carried out for immobilized persons and the results are tabulated in figure (8):

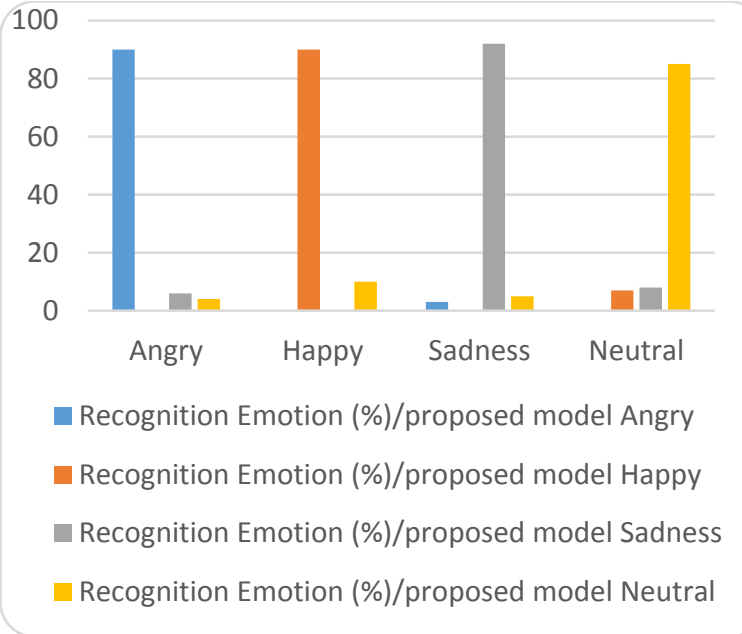

Fig.8. Recognition Rate (\%) of Emotions in the case of immobilized persons Doubly Truncated Gaussian Mixture Model

Table 1 shows the results of the proposed classifiers when Electroencephalography (EEG) signals are used.

Table 1. emotion recognition rates

\begin{tabular}{|c|c|c|c|c|}
\hline Name of the classifier & \multicolumn{4}{|c|}{ Double Truncated Gaussian Mixture } \\
Model \\
\hline $\begin{array}{c}\text { Data acquisition } \\
\text { method: }\end{array}$ & Happy & Sad & Angry & Neutral \\
\cline { 2 - 5 } Electroencephalography & 88 & 85 & 90 & 80 \\
\hline
\end{tabular}

\section{CONCLUSION}

The emotion recognition system develops using Double Truncated Gaussian mixture model with the parameter of the distribution is updated using EM Algorithm. Using this updated equations, the Probability Density Function are estimated. Basing on the Maximum Likelihood Function the emotions are identified. The results of the proposed system surpassed the results of conventional models. The proposed method can further be enhanced if speech signal is also added for emotion recognition.

\section{REFERENCE}

[1] John Atkinson, Daniel Campos (2016), "Improving BCIbased emotion recognition by combining EEG feature selection and kernel classifiers", Expert Systems with Applications Volume 47, 1 April 2016, Pages 35-41

[2] Jianhai Zhang, Ming Chen, Shaokai Zhao, Sanqing Hu, Zhiguo Shi and Yu Cao (2016)," ReliefF-Based EEG Sensor Selection Methods for Emotion Recognition", Sensors 2016, 16(10), 1558; doi:10.3390/s16101558

[3] Kahn, Branden," Electroencephalogram (EEG) signal processing, wave identification, and emotion recognition “, Scholar Works Open Access Repository, http://hdl.handle.net/10211.3/133547, 2015-01-21

[4] Alotaiby, T.; El-Samie, F.E.A.; Alshebeili, S.A.; Ahmad, I. "A review of channel selection algorithms for EEG signal processing”, EURASIP J. Adv. Signal Process. 2015. 
[5] J. Sirisha Devi, Y. Srinivas, "Automatic Speech Emotion and Speaker Verification based on Hybrid GMM and FFBNN", International Journal on Computational Sciences \& Applications (IJCSA) Vol.4, No.1, February 2014, ISSN: 2200-0011

[6] Jatupaiboon, N.; Pan-Ngum, S.; Israsena, P. Emotion classification using minimal EEG channels and frequency bands. In Proceedings of the International Joint Conference on Computer Science and Software Engineering, Khon Kaen, Thailand, 29-31 May 2013; pp. 21-24.

[7] Kim E. O. Min-Ki, Kim Miyoung and Kim S.-P. 2013 "A Review on the Computational Methods for Emotional State Estimation from the Human EEG", Computational and Mathematical Methods in Medicine 2013

[8] S. Koelstra, and others, DEAP: A Database for Emotion Analysis using Physiological Signals, IEEE Transactions on Affective Computing, 2011, pp. 1-15

[9] Khosrowabadi, R., Quek, H.C., Wahab, A. and Ang, K.K. (2010) "EEG-Based Emotion Recognition Using Self- Organizing Map for Boundary Detection", 20th International Conference on Pattern Recognition (ICPR), Istanbul,23-26 August 2010, 4242-4245.

[10] Reza Khosrowabadi \& Abdul Wahab bin Abdul Rahman(2010), "Classification of EEG correlates on emotion using features from Gaussian Mixtures of EEG Spectrogram", 3rd International Conference on ICT4M , issue no.31, pp.E102-E107.

[11] Petrantonakis P. C. and Hadjileontiadis L. J. 2010 Emotion recognition from EEG using higher order crossings." IEEE transactions on information technology in biomedicine : a publication of the IEEE Engineering in Medicine and Biology Society PP: 186-197

[12] Ling Wei,Yingjie Li, Jiping Ye (IEEE 2009)," Emotioninduced Higher Wavelet Entropy in the EEG with Depression during a Cognitive Task", International Conference of the IEEE EMBS, issue no.22, pp.50185021.

[13] Khalili, Z., Moradi, M.H.”: Emotion recognition system using brain and peripheral signals: Using correlation dimension to improve the results of EEG", in: Proceedings of International Joint Conference on Neural Networks. pp. 1571-1575 (2009)

[14] Sebastian Korb, Didier Grandjean \& Klaus Scherer (IEEE 2008), "Investigating the production of emotional facial expressions: a combined electroencephalographic (EEG) and electromyography (EMG) approach", issue no.19, pp.1-6.

[15] M. Murugappan, M.Rizon, R.Nagarajan, S.Yaacob, I.Zunaidi, and D.Hazry (2007), "EEG Feature Extraction for Classifying Emotions using FCM and FKM", International Journal of Computers and Communications, vol.1, issue no.30, pp.21-25.

[16] Ansari-Asl, K., Chanel, G. and Pun, T. (2007) "A Channel Selection Method for EEG Classification in Emotion Assessment Based on Synchronization Likelihood", EUSIPCO 2007, 15th European Signal Processing Conference, Poznan, 3-7 September 2007, 1241-1245.

\section{Authors' Profiles}

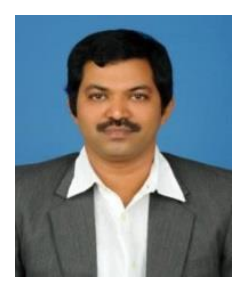

Dr. N. Murali Krishna was awarded B.Tech. from Computer Science \& Engineering department in the year 2002. $\mathrm{He}$ was awarded $\mathrm{M}$. Tech in Computer Science \& Engineering, in the year 2006. He was awarded doctorate in the year 2014.

His research interests include Human computer interaction and Software engineering. At present he is working as Associate Professor in Computer Science and Engineering Department, VITS, JNTU Hyderabad

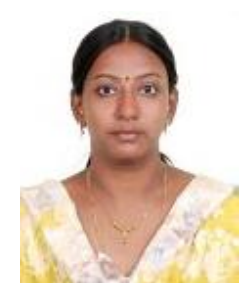

Dr. J Sirisha Devi was awarded B. Tech. in Computer Science and Engineering from Acharya Nagarjuna University -2003. She was awarded M. Tech. in Computer Science and Engineering from GITAM University, Visakhapatnam - 2010. She was awarded doctorate in the year 2016.

Her research interests include Human Computer Interaction and Software engineering and Data mining. At present she is working as Associate Professor in Computer Science and Engineering Department, JNTU Kakinada.

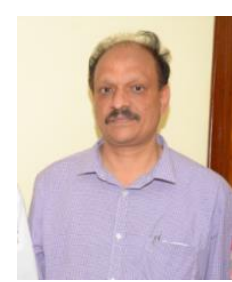

Dr. Srinivas Yarramalle was awarded M.Tech. in Computer Science and Engineering department in the year 1999. He was awarded doctorate in the year 2008.

His research interests include Human computer interaction, image processing and Software engineering. At present he is working as HOD in the Department of Information Technology, GIT, GITAM University, Visakhapatnam

How to cite this paper: N Murali Krishna, J Sirisha Devi, Srinivas Yarramalle,"A Novel Approach for Effective Emotion Recognition Using Double Truncated Gaussian Mixture Model and EEG", International Journal of Intelligent Systems and Applications(IJISA), Vol.9, No.6, pp.33-42, 2017. DOI: 10.5815/ijisa.2017.06.04 